1 Universidade Estadual de Londrina (UEL) Departamento de Saúde Coletiva - Londrina (PR), Brasil.

marsellecarvalho@gmail.com

2 Universidade Estadual de Londrina (UEL),

Departamento de Saúde

Coletiva - Londrina (PR),

Brasil.

esterdallacosta@gmail.com

3 Universidade Estadual de Londrina (UEL),

Departamento de Saúde Coletiva - Londrina (PR),

Brasil.

sakai.marcia@gmail.com

4 Universidade Estadual de Londrina (UEL), Departamento de Saúde Coletiva - Londrina (PR), Brasil.

reginagil75@gmail.com

5 Universidade Federal de Santa Catarina (UFSC), Departamento de Ciências Farmacêuticas Florianópolis (SC), Brasil. silvana.nair@hotmail.com

\section{Expansão e diversificação da força de trabalho de nível superior nas Unidades Básicas de Saúde no Brasil, 2008 - 2013}

\author{
Expansion and diversification of university level workforce in \\ Primary Health Care Units Brazil:2008 - 2013
}

Marselle Nobre de Carvalho', Ester Massae Okamoto Dalla Costaㄹ, Marcia Hiromi Sakai ${ }^{\mathbf{3}}$, Célia

Regina Rodrigues Gil4, Silvana Nair Leite ${ }^{\mathbf{5}}$

RESUMO Este artigo objetiva identificar os trabalhadores de nível superior cadastrados nas Unidades Básicas de Saúde (UBS) entre 2008 e 2013, visando contribuir com o debate sobre o planejamento da força de trabalho na Atenção Básica em saúde. Trata-se de estudo descritivo, retrospectivo e de abordagem quantitativa. Embora médicos, enfermeiros e cirurgiões-dentistas componham as ocupações de nível superior tradicionalmente hegemônicas nas UBS, constatou-se a ampliação da participação de outras categorias profissionais, o que se atribui à implantação de equipes do Núcleo de Apoio à Saúde da Família em todo o País.

PALAVRAS-CHAVE Recursos humanos em saúde; Centros de saúde; Atenção Primária à Saúde.

ABSTRACT This article aims to identify the university level workforce enrolled in the Primary Health Care Units (UBS) between 2008 and 2013, to contribute to the debate on the primary health care workforce planning. It is a descriptive and retrospective study of quantitative approach. Although physicians, nurses and dentists comprise the traditionally hegemonic university level workforce in UBS, there was observed an increasing participation of other professional categories, which may be due to the implementation of health supporting teams throughout the Country.

KEYWORDS Health manpower; Health centers; Primary Health Care. 


\section{Introdução}

A Atenção Básica (AB) é ordenadora e considerada porta preferencial de acesso da população ao Sistema Único de Saúde (SUS) e às Unidades Básicas de Saúde (UBS); com ou sem saúde da família, são indispensáveis à realização das ações de saúde nos municípios e no Distrito Federal. As UBS devem disponibilizar equipe multiprofissional composta por médico, enfermeiro, cirurgião dentista, auxiliar de saúde bucal ou técnico em saúde bucal, auxiliar de enfermagem ou técnico de enfermagem e agente comunitário de saúde, dentre outros (BRASIL, 2012B; CARVALHO ET AL., 2014).

Segundo Machado, Oliveira e Moyses (2011), o período de 2005 a 2010 foi marcado pela expansão do SUS, o que resultou no aumento da participação dos municípios e na relativa queda da União, no total de empregos públicos em saúde e no aumento do grau de escolaridade dos trabalhadores.

Apesar disso, o Ministério da Saúde reconhece que a formação e a distribuição dos trabalhadores em saúde na rede de serviços tem sido um grande desafio para a consolidação da AB. Afinal, a possibilidade de mudanças e melhorias dos serviços de saúde se dá a partir das relações que se estabelecem entre trabalhadores e usuários, tanto entre si como de uns para os outros (RIZZOTO ET AL., 2014).

Embora não haja estatísticas organizadas sobre recursos humanos no mundo, Giovanella et al. (2015) informa que existe consenso entre os gestores sobre a oferta insuficiente quantitativa e qualitativamente em diversos países, especialmente na América do Sul, e uma estratégia para superação desse cenário tem sido a cooperação entre os países, sobretudo aqueles que possuem trabalhadores com formação em $\mathrm{AB}$, como é o caso de Cuba. Além da disponibilidade numérica, a disponibilidade para atuar na saúde da família é outro problema relevante para a $\mathrm{AB}$ no Brasil (CAVALCANTI; OLIVEIRA NETO; SOUSA, 2015).

Ao longo da última década, na tentativa de modificar o cenário de escassez e distribuição desigual de recursos humanos nos serviços de saúde no Brasil, o governo federal tem implementado estratégias, programas e projetos dirigidos à reestruturação da $\mathrm{AB}$, como, por exemplo, a Estratégia Saúde da Família (ESF), o Núcleo de Apoio à Saúde da Família (Nasf), o Programa Nacional para Melhoria do Acesso e da Qualidade da Atenção Básica (PMAQ-AB), o Programa de Valorização do Profissional de Atenção Básica (Provab) e, mais recentemente, o Mais Médicos (LOPES, 2013; RIZZOTO ET AL., 2014).

Nesse contexto, este artigo tem como objetivo identificar os trabalhadores de nível superior cadastrados nas UBS entre 2008 e 2013, visando à contribuição para o debate sobre o planejamento e dimensionamento da força de trabalho no SUS.

\section{Método}

Abordaram-se, no estudo, as catorze profissões de saúde de interesse para o SUS listadas na Resolução no 287, de 08 de outubro de 1998, do Conselho Nacional de Saúde (CNS).

A pesquisa se circunscreveu à UBS, definida como unidade destinada à relação de atendimentos de $\mathrm{AB}$ e integral a uma população, de forma programada ou não, podendo oferecer assistência odontológica e de outros profissionais de nível superior, com tipologia e equipes de saúde bem definidas (BRASIL, 2012B).

O Cadastro Nacional de Estabelecimentos da Saúde (CNES), utilizado como fonte dos dados, disponibiliza informações dos postos de trabalho, organizados a partir da Classificação Brasileira de Ocupações (CBO), bem como dos trabalhadores, sendo possível determinar a quantidade de vínculos de trabalho de cada trabalhador. Estão incluídos todos os trabalhadores dos estabelecimentos de saúde, quais sejam, os de nível superior, médio e fundamental relacionados diretamente à assistência, bem como os de áreas administrativas e de gerenciamento do estabelecimento. Essas informações podem ser 
analisadas segundo diferentes características dos estabelecimentos de saúde cadastrados (SALA; CARRO; SEIXAS, 2010).

Uma das limitações do uso da base CNES diz respeito à sua abrangência, dado que não há obrigatoriedade de inclusão em seu universo dos serviços de apoio à gestão, redundando na não inclusão de todos os profissionais de nível administrativo, vinculados ou não ao SUS. A outra limitação se refere aos estabelecimentos estritamente privados que ainda não estão totalmente cadastrados na base CNES, em especial os ambulatórios de média complexidade, a despeito de todo o esforço da Secretaria de Atenção à Saúde (SAS) e dos gestores para captação dessas informações (BRASIL, 2007).

Os dados foram coletados no mês de outubro de 2014, ratificados em dezembro de 2014 e fevereiro de 2015, no banco de dados on-line disponível no portal do Departamento de Informática do Ministério da Saúde, acessado pelo endereço eletrônico www.datasus.gov.br. Realizou-se sua tabulação com o auxílio do programa Microsoft Office Excel ${ }^{\circledR}$, de onde se extraíram basicamente frequências e percentuais.

\section{A expansão da $\mathrm{AB}$}

A década de 1990 foi marcada pela implantação do SUS e expansão do mercado de trabalho no setor de saúde no Brasil, sobretudo na esfera municipal. Em 1999, os municípios detinham $92 \%$ dos estabelecimentos públicos do País, enquanto a União e os estados, apenas $2 \%$ e $6 \%$, respectivamente. O emprego público no nível municipal cresceu $700 \%$ entre 1992 e 2005 (VARELLA; PIERANTONI, 2008).
Além do processo de descentralização da gestão, o mercado de trabalho em saúde foi positivamente impactado pela reestruturação do modelo de atenção à saúde, com a criação dos Programas de Agentes Comunitários de Saúde (Pacs), em 1991, e Programa Saúde da Família (PSF), em 1994, pelo Ministério da Saúde (BRASIL, 2006).

$A \mathrm{AB}$, entendida no Brasil, por muitos autores e especialmente pelos formuladores de políticas de saúde, como termo correlato à Atenção Primária à Saúde (APS), ganhou centralidade no modelo de atenção, deixando o PSF de ser um programa para se tornar uma estratégia prioritária para a reorganização do SUS. Nesse contexto, o Ministério da Saúde publicou a Política Nacional de Atenção Básica (PNAB), em 2006, por meio da qual, passou a adotar o termo Estratégia de Saúde da Família (ESF), que direciona o cuidado em saúde para o indivíduo e sua família (BRASIL, 2006; GIL, 2006).

Segundo a PNAB, a UBS é o estabelecimento fundamental da $\mathrm{AB}$ e pode ter ou não Equipe de Saúde da Família (EqSF). A EqSF é multiprofissional, composta por um enfermeiro, um médico generalista ou de família, um auxiliar de enfermagem e agentes comunitários de saúde (BRASIL, 2012A).

Entre 1981 e 2008, o número de pessoas que procuraram atendimento na $\mathrm{AB}$ aumentou cerca de $450 \%$, o que se deve ao crescimento do número de unidades de $\mathrm{AB}$ e ao tamanho da força de trabalho do setor da saúde (PAIM ET AL., 2011).

$O$ crescimento da $\mathrm{AB}$ continuou entre 2008 e 2013, especialmente pela expansão da ESF e pela implantação do Nasf, conforme mostra a tabela 1. 
Tabela 1. Evolução da rede assistencial e equipes da Atenção Básica do SUS. Brasil 2008 - 2013

\begin{tabular}{lcccc}
\hline Rede Assistencial & Dez/2008 & Dez/2013 & Variação(n) & Crescimento (\%) \\
\hline Unidades Básicas de Saúde & 30.157 & 34.009 & 3.852 & 13 \\
Equipes de SF implantadas & 29.346 & 34.715 & 5.369 & 18 \\
Equipes de Nasf implantadas & - & 2.767 & 2.767 & - \\
\hline
\end{tabular}

Fonte: Ministério da Saúde - Cadastro Nacional dos Estabelecimentos de Saúde do Brasil - CNES (BRASIL, 2014).

SF = Saúde da Família; Nasf = Núcleo de Apoio à Saúde da Família .

Em 2015, a ESF atingiu uma cobertura superior a $60 \%$ da população, com mais de 40 mil EqSF implantadas, garantindo assistência para aproximadamente 130 milhões de brasileiros (BRASIL, 2014; BRASIL, 2016B).

Com a finalidade de ampliar as ações da
AB, o Ministério da Saúde criou os Nasfs, equipes compostas por profissionais de diferentes áreas de conhecimento que atuam em parceria com os profissionais das EqSF nas modalidades Nasf 1, 2 e 3, conforme expõe o quadro 1.

Quadro 1. Modalidades de Nasf de acordo com o número de equipes vinculadas e somatório das cargas horárias.

\begin{tabular}{|c|c|c|}
\hline Modalidades & № de equipes vinculadas & Somatório das cargas horárias profissionais \\
\hline Nasf 1 & $\begin{array}{r}5 \text { a } 9 \text { EqSF ou EqAB para populações } \\
\text { específicas (EqCR, EqSFR e EqSFF) }\end{array}$ & $\begin{array}{r}\text { Mínimo } 200 \text { horas semanais; } \\
\text { Cada ocupação deve ter no mínimo } 20 \text { h e no máximo } \\
\text { 80h de carga horária semanal; }\end{array}$ \\
\hline Nasf 2 & $\begin{array}{r}3 \text { a } 4 \text { EqSF e/ou EqAB para populações } \\
\text { específicas (EqCR, EqSFR e EqSFF) }\end{array}$ & $\begin{array}{r}\text { Mínimo } 120 \text { horas semanais; } \\
\text { Cada ocupação deve ter no mínimo } 20 \text { h e no máximo } \\
\text { 40h de carga horária semanal; }\end{array}$ \\
\hline Nasf 3 & $\begin{array}{r}1 \text { a } 2 \text { EqSF e/ou EqAB para populações } \\
\text { específicas (EqCR, EqSFR e EqSFF) }\end{array}$ & $\begin{array}{r}\text { Mínimo } 80 \text { horas semanais; } \\
\text { Cada ocupação deve ter no mínimo } 20 \text { h e no máximo } \\
\text { 40h de carga horária semanal; }\end{array}$ \\
\hline
\end{tabular}

Fonte: Departamento de Atenção Básica do Ministério da Saúde. Extraído de http://dab.saude.gov.br/portaldab/ape_nasf.php. (BRASIL, 2016A). EqSF = Equipe de Saúde da Família; EqAB = Equipe de Atenção Básica; EqCR = Equipes de Consultório de Rua; EqSFR = Equipe de Saúde da Família Rural; EqSFF = Equipe de Saúde da Família Fluvial.

A composição de cada Nasf é definida pelo gestor municipal segundo critérios de prioridade identificados a partir dos dados epidemiológicos, das necessidades locais e das equipes de saúde a serem apoiadas. Podem compor equipes Nasf as seguintes ocupações: assistente social, profissional/professor de educação física, farmacêutico, fisioterapeuta, fonoaudiólogo, médico acupunturista, ginecologista/ obstetra, homeopata, pediatra, psiquiatra, geriatra, internista/clínica médica, nutricionista, psicólogo, terapeuta ocupacional, médico do trabalho, médico veterinário, arte educador e sanitarista (BRASIL, 2008; BRASIL, 2012A). 


\section{A diversificação da força de trabalho de nível superior}

Entre 2008 e 2013, a população do País cresceu a uma taxa de $6 \%$ no período, as UBS cadastradas no CNES, de $12 \%$ no período, e o número de profissionais de nível superior cadastrados nesses estabelecimentos, de $24 \%$ no período, o que significa expansão da capacidade instalada, aumento do número de postos de trabalho e possibilidade de ampliação da força de trabalho de todas as categorias de profissionais de nível superior nesse tipo de estabelecimento de saúde no âmbito do SUS.

As ocupações de nível superior cadastradas nas UBS apresentaram incremento de 31.524 trabalhadores, crescendo em torno de $16 \%$ no período estudado. Embora médicos, enfermeiros e cirurgiões dentistas sejam as profissões hegemônicas nesses serviços de saúde, ocupando, respectivamente, 39,7\%, $23,9 \%$ e $18,4 \%$ dos postos de trabalho, mostraram as menores taxas de crescimento no período de 2008 a 2013 (tabela 2).

Tabela 2. Ocupações de nível superior cadastradas nos estabelecimentos do tipo Centro de Saúde/Unidade Básica de Saúde. Brasil 2008 - 2013

\begin{tabular}{|c|c|c|c|c|c|c|}
\hline \multirow[t]{2}{*}{ Ocupação CBO 2002} & \multicolumn{2}{|l|}{ Dez/08 } & \multicolumn{2}{|l|}{$\mathrm{Dez} / 13$} & \multirow[t]{2}{*}{ Variação (n) } & \multirow{2}{*}{$\begin{array}{l}\text { Crescimento } \\
(\%)\end{array}$} \\
\hline & $\mathbf{N}$ & $\%$ & $\mathbf{N}$ & $\%$ & & \\
\hline \multicolumn{7}{|l|}{ Equipe SF } \\
\hline Enfermeiro & 44.723 & 22,3 & 55.468 & 23,9 & 10.745 & 24 \\
\hline Médico & 89.609 & 44,7 & 92.290 & 39,7 & 2.681 & 03 \\
\hline Cirurgião-Dentista & 39.392 & 19,6 & 42.634 & 18,4 & 3.242 & 08 \\
\hline \multicolumn{7}{|l|}{ Equipe Nasf } \\
\hline Assistente Social & 5.193 & 2,6 & 6.754 & 2,9 & 1.561 & 30 \\
\hline Farmacêutico* & 2.771 & 1,4 & 4.860 & 2,1 & 2.089 & 75 \\
\hline Fisioterapeuta & 5.188 & 2,6 & 9.248 & 4.0 & 4.060 & 78 \\
\hline Fonoaudiólogo & 2.179 & 1,1 & 3.377 & 1,5 & 1.198 & 55 \\
\hline Médico Veterinário & 565 & 0,3 & 273 & 0,1 & -292 & -52 \\
\hline Nutricionista & 3.306 & 1,6 & 6,055 & 2,6 & 2,749 & 83 \\
\hline Prof. Ed. Física** & 98 & - & 240 & 0,1 & 142 & 145 \\
\hline Psicólogo & 6.516 & 3,2 & 9.303 & 4,0 & 2.787 & 43 \\
\hline Terapeuta Ocupacional & 695 & 0,3 & 1.244 & 0,5 & 549 & 79 \\
\hline
\end{tabular}


Tabela 2 (cont.)

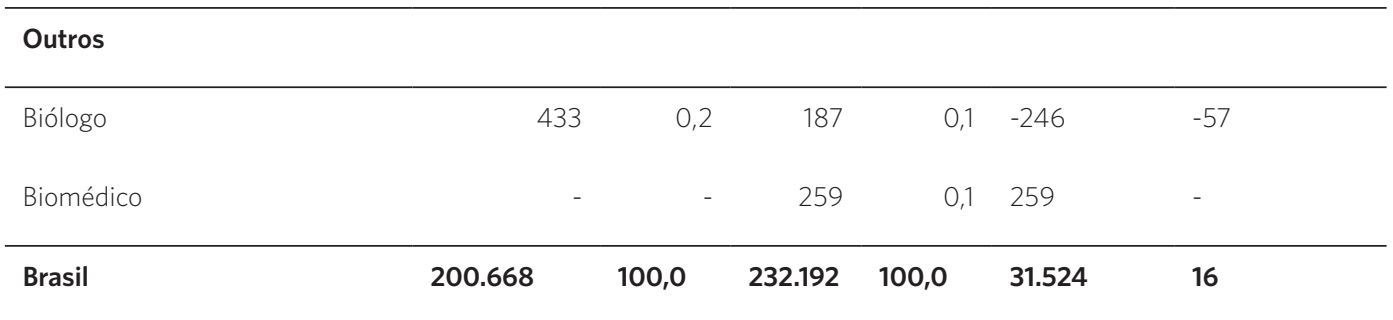

Fonte: Ministério da Saúde - Cadastro Nacional dos Estabelecimentos de Saúde do Brasil - CNES (BRASIL, 2014).

*Excluído o farmacêutico-bioquímico.

** Professor de Educação Física Ensino Fundamental, Médio e Superior.

SF = Saúde da Família; Nasf = Núcleo de Apoio à Saúde da Família.

Já as categorias profissionais de menor participação na composição da força de trabalho nas UBS, com percentuais em torno de 0,1 a $3 \%$, cresceram entre $50 \%$ a $150 \%$, como é o caso de fonoaudiólogos (55\%), farmacêuticos (75\%), fisioterapeutas (78\%), terapeutas ocupacionais (79\%), nutricionistas (83\%) e professores/profissionais de educação física (145\%). Algumas profissões, no entanto, apresentaram retração, como, por exemplo, a medicina veterinária (tabela 2 ).

Embora os médicos ainda sejam a categoria hegemônica do setor saúde e tenham apresentado expressivo crescimento no número de postos de trabalho nos anos 1970 e 1980, a composição interna da força de trabalho, nos anos 1990 e 2000, diversificou-se, ficando evidente a inclusão de novas categorias profissionais de nível superior (GIRARDI; CARVALHO, 2002),

O estudo de Nunes et al. (2015), realizado em municípios de pequeno porte no estado do Paraná, também identificou a presença marcante de equipes multiprofissionais nas UBS. Além dos profissionais convencionais das EqSF, havia fisioterapeutas, médicos veterinários, psicólogos, nutricionistas, fonoaudiólogos e assistentes sociais, dentre outros. Segundo esses autores:

Esses profissionais têm potencial para exercer e apoiar ações de promoção, proteção e vigilância à saúde. Assim, a diversidade de categorias profissionais sugere, ainda que incipientemente, a perspectiva de ampliar a abrangência e o escopo das ações da $A B$, bem como o aumento de sua resolutividade. (NUNES ET AL., 2015, P. 38).

Entendendo a PNAB como referência e considerando a equipe convencional da ESF, entre 2008 e 2013, as categorias profissionais que compõem o Nasf apresentaram crescimento superior aos enfermeiros, médicos e cirurgiões dentistas.

Algumas categorias cresceram taxas acima de $90 \%$, como é o caso dos professores/profissionais de educação física, fisioterapeutas, nutricionistas e terapeutas ocupacionais. Fonoaudiólogos e psicólogos cresceram em torno de $50 \%$. No caso dos psicólogos, é possível que o aumento tenha se dado também em razão da criação dos consultórios de rua, cujas ações são desenvolvidas na rua, em instalações específicas, unidades móveis e nas instalações das UBS do território onde estão atuando. Agem sempre articuladamente, desenvolvendo ações em parceria com as demais equipes de $\mathrm{AB}$ do território (UBS e Nasf), dos Centros de Atenção Psicossocial (Caps), da Rede de Urgência e dos serviços e instituições componentes do Sistema Único de Assistência Social (BRASIL, 2012). 


\section{Conclusão}

O termo força de trabalho em saúde, geralmente, é empregado com referência aos profissionais de saúde - médicos, enfermeiros, psicólogos, dentistas, dentre outros - mas há também uma definição ampliada da força de trabalho em saúde que inclui a força de trabalho não profissional, como líderes comunitários, voluntários, pacientes que exercem autocuidado e familiares que prestam cuidados à saúde. Neste estudo, o termo diz respeito aos trabalhadores com formação superior e cadastrados no CNES.

Os resultados mostram que, gradativamente, outras profissões de saúde têm ocupado espaço na $\mathrm{AB}$ de saúde, ponto positivo a favor da integralidade do cuidado e da resolutividade desse ponto de atenção. Evidencia-se que o trabalho em saúde tem sido desenvolvido, cada vez mais, por equipes multiprofissionais e multidisciplinares, o que se deve à criação e implementação da ESF e, especialmente, à implantação do Nasf, em 2008.

O objetivo do planejamento da força de trabalho em saúde é encontrar o equilíbrio entre a composição, a distribuição e o número de trabalhadores para que as ações dos serviços resultem em efetiva melhoria dos níveis de saúde das comunidades e populações.

Nesse contexto, se destaca a importância dos Observatórios de Recursos Humanos em Saúde, tanto nacional quanto regionais, por oportunizarem a realização de estudos de monitoramento e avaliação do impacto da diversificação da força de trabalho na $A B$. Cabe destacar que a melhoria dos indicadores de saúde, sobretudo na prevenção de agravos, redução da hospitalização e melhoria da qualidade de vida requerem, sobretudo, ações produzidas pela força de trabalho em saúde.

\section{Referências}

BRASIL. Ministério da Saúde. Departamento de Informática do Ministério da Saúde. 2014. Disponível em: <http://datasus.saude.gov.br>. Acesso em: 10 mar. 2014.

Ministério de Saúde. Indicadores de gestão do trabalho em saúde: material de apoio para o Programa de Qualificação e Estruturação da Gestão do Trabalho e da Educação no SUS - ProgeSUS. Brasília, DF: Ministério da Saúde, 2007. Disponível em: <https://www.nescon.medicina.ufmg.br/biblioteca/ imagem/3062.pdf>. Acesso em: 10 mar. 2014.

Ministério da Saúde. Portal da Saúde. 2016a.
Disponível em: <http://dab.saude.gov.br/portaldab/ ape_nasf.php>. Acesso em: 18 abr. 2016.

Ministério da Saúde. Portal da Saúde. 2016b. Disponível em: <http://dab.saude.gov.br/dab/historico_ cobertura_sf/historico_cobertura_sf_relatorio.php>. Acesso em: 16 maio 2016.

Ministério da Saúde. Portaria n ${ }^{\circ} 154$ de 24 de janeiro de 2008. Cria os Núcleos de Apoio à Saúde da Família - NASF. Diário Oficial [da] União. Brasília, DF, 24 jan. 2008. Disponível em: <http://dtr2001.saude. gov.br/sas/PORTARIAS/Port2008/GM/GM-154.htm>. Acesso em: 10 mar. 2014. 
Ministério da Saúde. Portaria n ${ }^{\circ} 3.124$, de 28 de dezembro de 2012. Redefine os parâmetros de vinculação dos Núcleos de Apoio à Saúde da Família (NASF) Modalidades 1 e 2 às Equipes Saúde da Família e/ou Equipes de Atenção Básica para populações específicas, cria a Modalidade NASF 3, e dá outras providências. Diário Oficial [da] União, Brasília, DF, 28 dez. 2012a. Disponível em: <http://bvsms.saude.gov.br/ bvs/saudelegis/gm/2012/prt3124_28_12_2012.html>. Acesso em: 15 jul. 2015.

. Ministério da Saúde. Secretaria de Atenção à Saúde. Departamento de Atenção Básica. Política Nacional de Atenção Básica. Brasília, DF: Ministério da Saúde, 2012b. Disponível em: <http://189.28.128.100/ dab/docs/publicacoes/geral/pnab.pdf > . Acesso em: 15 jul. 2015

Ministério da Saúde. Secretaria de Atenção à Saúde. Departamento de Atenção Básica. Saúde da família no Brasil: uma análise de indicadores selecionados: 1998-2004. Brasília, DF: Ministério da Saúde, 2006. Disponível em: <http://189.28.128.100/ dab/docs/publicacoes/informes/psfinfo37.pdf > . Acesso em: 10 mar. 2014.

CARVALHO, B. G. et al. Gerência de unidade básica de saúde em municípios de diferentes portes: perfil e instrumentos gerenciais utilizados. Revista da Escola de Enfermagem da USP, v. 48, n. 5, 2014. Disponível em: $<$ http://www.scielo.br/pdf/reeusp/v48n5/pt_00806234-reeusp-48-05-907.pdf>. Acesso em: 10 mar. 2014.

CAVALCANTI, P. C. S.; OLIVEIRANETO, A.V.; SOUSA, M. F. Quais são os desafios para a qualificação da Atenção Básica na visão dos gestores municipais? Saúde em Debate. Rio de Janeiro, v. 39, n. 105, p. 323336, abr./jun. 2015.

GIL, C. R. R. Atenção primária, atenção básica e saúde da família: sinergias e singularidades do contexto brasileiro. Cadernos de Saúde Pública, Rio de Janeiro, v. 22, n. 6, p. 1171-81, jun. 2006.

GIOVANELLA, L. et al. Panorama de la Atención Primaria de Salud en Suramérica: concepciones, componentes y desafios. Saúde em Debate. Rio de
Janeiro, v. 39, n. 105, p. 300-322, abr./jun. 2015.

GIRARDI, S. N. O perfil do "emprego" em saúde no Brasil. Cadernos de Saúde Pública, Rio de Janeiro, v. 2, n. 4, dez. 1986.

GIRARDI, S. N.; CARVALHO, C. L. Configurações do mercado de trabalho dos assalariados em saúde no Brasil. Formação, Brasília, DF, v. 2, n. 6 p. 15-36, 2002.

LOPES, L. R. S. Demografia médica: provimento e fixação de médicos em áreas de maior vulnerabilidade. 2013. 61 f. Trabalho de Conclusão de Curso (Curso de Altos Estudos de Política e Estratégia) - Escola Superior de Guerra, Rio de Janeiro, 2013. Disponível em: <http://www.esg.br/images/Monografias/2013/ LOPES.pdf $>$. Acesso em: 10 mar. 2014.

\section{MACHADO, M. H.; OLIVEIRA, E. S.; MOYSES,}

N. M. N. Tendências do Mercado de Trabalho em Saúde no Brasil. In: PIERANTONI, C.; POZ, M. R. D.; FRANÇA, T. (Org.). O Trabalho em Saúde: abordagens quantitativas e qualitativas. Rio de Janeiro: CEPESC/ UERJ, 2011 p. 103-116. Disponível em: < http://www. ensp.fiocruz.br/observarh/arquivos/Tendencias\%25 20de\%2520Mercado\%2520de\%2520Trabalho.pdf>. Acesso em: 10 mar. 2014.

NUNES, E. F. P. A. et al. Força de trabalho em saúde na Atenção Básica em Municípios de Pequeno Porte do Paraná. Saúde em Debate, v. 39, n. 104. Rio de janeiro, jan./mar. 2015.

PAIM, J. et al. The Brazilian health system: history, advances, and challenges. Lancet, Londres. v. 377, maio 2011. Disponível em: <http://www6.ensp.fiocruz.br/ repositorio/sites/default/files/arquivos/The\%20 Brazilian_Celia\%20Almeida_2011.pdf>. Acesso em: 15 dez. 2013.

RIZZOTO, M. L. F. et al. Força de trabalho e gestão do trabalho em saúde: revelações da Avaliação Externa do Programa Nacional de Melhoria do Acesso e da Qualidade da Atenção Básica no Paraná. Saúde em Debate, Rio de Janeiro, v. 38, número especial, p. 237251, out. 2014. 
SALA, A.; CARRO, A. R. L.; SEIXAS, P. H. A.

Comparação entre as bases de dados relativas à força de trabalho em saúde no Estado de São Paulo. Boletim eletrônico do grupo técnico de avaliação e informações de saúde, Rio de Janeiro, 2010. p. 1-7. Disponível em: <http://portal.saude.sp.gov.br/resources/ses/perfil/ gestor/homepage//gais-informa/gais_jornal_4.pdf>. Acesso em: 15 dez. 2013.

VARELLA, T. C.; PIERANTONI, C. R. Mercado de trabalho: revendo conceitos e aproximando o campo da saúde. A década de 90 em destaque. Physis: Revista de Saúde Coletiva, Rio de Janeiro, v. 18, n. 3, p. 521-544, set. 2008. Disponível em: <http://www. scielo.br/scielo.php?script=sci_arttext\&pid=S0103$73312008000300009 \& \operatorname{lng}=\mathrm{en} \& \mathrm{nrm}=\mathrm{iso}>$. Acesso em: 10 dez. 2013.

Recebido para publicação em outubro de 2015

Versão final em abril de 2016

Conflito de interesses: inexistente

Suporte financeiro: não houve 